\title{
Method for Water Vapor Profile Retievals by Means of Minimizing Difference Between Estimated and Actual Brightness Temperatures Derived from AIRS data and Radiative Transfer Model
}

\author{
Kohei Arai ${ }^{1}$ \\ (Graduate School of Science and Engineering) \\ Saga University \\ Saga City, Japan
}

\begin{abstract}
Method for water vapor profile retrievals by means of minimizing difference between estimated and actual brightness temperatures derived from AIRS data and radiative transfer model is proposed. Initial value is determined by linearized radiative transfer equation. It is found that this initial value determination method makes improvement of estimation accuracy together with reducing convergence time.
\end{abstract}

Keywords-infrared sounder; non-linear optimization method; linearized inversion.

\section{INTRODUCTION}

Air-temperature and water vapor profiles are used to be estimated with Infrared Sounder data [1]. One of the problems on retrieving vertical profiles is its retrieving accuracy. In particular, estimation accuracy of air-temperature and water vapor at tropopause altitude is not good enough because there are gradient changes of air-temperature and water vapor profile in the tropopause so that observed radiance at the specific channels are not changed for the altitude.

In order to estimate air-temperature and water vapor, least square based method is typically used. In the process, Root Mean Square: RMS difference between observed radiance and calculated radiance with the designated physical parameters are minimized. Then the designated physical parameters including air-temperature and water vapor at the minimum RMS difference are solutions.

Typically, Newton-Raphson method which gives one of local minima is used for minimization of RMS difference. Newton-Raphson needs first and second order derivatives, Jacobean and Hessian at around the current solution. It is not easy to formularize these derivatives analytically. The proposed method is based on Levenberg Marquardt: LM of non-linear least square method. It uses numerically calculated first and second order derivatives instead of analytical based derivatives. Namely, these derivatives can be calculated with radiative transfer model based radiance calculations. At around the current solution in the solution space, directional derivatives are calculated with the radiative transfer model.

The proposed method is validated for air-temperature and water vapor profile retrievals with Infrared: IR sounder data derived from Atmospheric Infrared Sounder:/AIRS onboard AQUA satellite [2]-[7]. A comparison of retrieving accuracy between Newton-Raphson method and the proposed method based on LM method [8] is made in order to demonstrate an effectiveness of the proposed method in terms of estimation accuracy in particular for the altitude of tropopause [9]. Global Data Assimilation System: GDAS data of assimilation model derived 1 degree mesh data is used as truth data of airtemperature and water vapor profiles. The experimental data show that the proposed method is superior to the conventional Newton-Raphson method.

The following section describes proposed method for water vapor profile retrievals followed by experiments. Then finally, conclusion and some discussions are described.

\section{PROPOSED METHOD}

\section{A. Radiative Transfer Equation}

Radiative transfer equation is expressed with equation (1).

$$
R v=\left(I_{0}\right)_{v} \tau_{v}\left(z_{0}\right)+\int_{z_{0}}^{\infty} B v\{T(z)\} K_{v}(z) d z
$$

where vdenotes wave number $(\mathrm{cm}-1)$, and

$\mathrm{Rv}$ : at sensor brightness temperature

(I0) $v$ : brightness temperature of ground surface

$\tau \quad v(\mathrm{z} 0):$ total column atmospheric transmittance

$\mathrm{B}\{\mathrm{T}(\mathrm{z})\} \mathrm{v}$ : Planckian function of air temperature at the altitude of $\mathrm{z}$

$\mathrm{K} v(\mathrm{z})$ : atmospheric transmittance at the altitude of $\mathrm{z}$

This equation (1) can be linearized as follows,

$$
R=B K
$$

where the number of unknown variables and the number of given equations are same. Therefore, it can be solved relatively easily. This solution from linear inversion provides initial value of the steepest descent method. Without this initial value, steepest descent method falls in one of local minima easily. 


\section{B. Water Vapor Profile Retrival Method}

For instance, it can be solved based on steepest descent method as shown in equation (3)

$$
R-R_{0}=\frac{\partial R}{\partial q}\left(q-q_{0}\right)
$$

Also, it is possible to estimate water vapor profile to minimize the following covariance matrix of error,

$$
\hat{x}=x_{a}+\left(A^{T} S_{\varepsilon}^{-1} A+S_{a}^{-1}\right)^{-1} A^{T} S_{\varepsilon}^{-1}\left(R-R_{a}\right)
$$

where

$x_{a}$ : Designated variable matrix

$\hat{x}:$ Variable matrix for estimation

A: Jacobian Matrix

$S_{\varepsilon}$ : Covariance matrix for measurement error

$R$ : Observed brightness temperature

$R_{a}$ : Estimated brightness temperature

Covariance matrix can be defined as equation (5).

$$
S_{i j}=\varepsilon\left(x_{i}-\hat{x}_{i}\right)\left(x_{j}-\hat{x}_{j}\right)^{T}
$$

Jacobian Matrix can be expressed in equation (6).

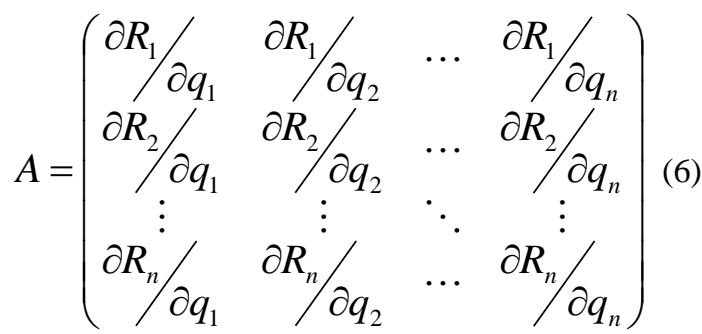

C. Steepest Descent Method (Non-linear optimization method) Steepest descent method can be represented in equation (7).

$$
q_{k}=q_{k-1}+\alpha_{k} g_{k}
$$

where

$q_{k}$ : estimated value at the iteration number $\mathrm{k}$

$g$ : updating vector

$\alpha$ : step width
Estimated value can be updated with the direction of $g$ and with step size of $\alpha$. Then estimation process is converged at one of local minima, not global optimum solution. This learning or updating process can be illustrated as shown in Figure 1. Initial value is derived from the linear inversion, $\mathrm{K}=\mathrm{B}-1 \mathrm{R}$.

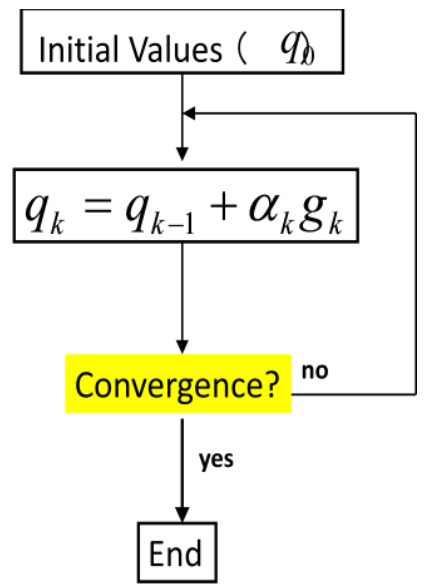

Figure 1. Process flow of steepest descent method

\section{EXPERIMENTS}

\section{A. AIRS Channels Used for Estimation of Water Vapor Profile}

AIRS channels used for estimation of water vapor profile and the corresponding wave numbers are shown in Table 1.

TABLE I. AIRS CHANNELS USED FOR ESTIMATION OF WATER VAPOR PROFILE AND THE CORRESPONDING WAVE NUMBERS

\begin{tabular}{|l|l|l|l|l|l|l|l|l|l|}
\hline Wave Number & 1478 & 1483 & 1508 & 1514 & 1519 & 1541 & 1544 & 1558 & 1585 \\
\hline AIRS channel & 1684 & 1692 & 1731 & 1740 & 1748 & 1761 & 1765 & 1786 & 1824 \\
\hline
\end{tabular}

\section{B. AIRS Data Used}

AIRS data of Mexican gulf (Latitude: 20 degree North, Longitude: 92 degree West) which is acquired at 18:00 on November 16 in 2002 is used. Intensive study area is shown in Figure 2. Level 1B brightness temperature is shown in Figure 3. On the other hands, MODTRAN of radiative transfer code is used for estimation of at sensor brightness temperature. Mid. Latitude Winter of atmospheric model is assumed. Also carbon dioxide mixing ratio and total column water vapor as well as ozone is set at default values.

\section{Weighting Function and Brightness Temperature}

One of the examples of at sensor brightness temperature of AIRS (Water vapor absorption channels of the wave number ranges from 1460 to $1620 \mathrm{~cm}-1$ ) is shown in Figure 4. Weighting function which corresponds to the water vapor absorption channels of wave number are shown in Figure 5. 


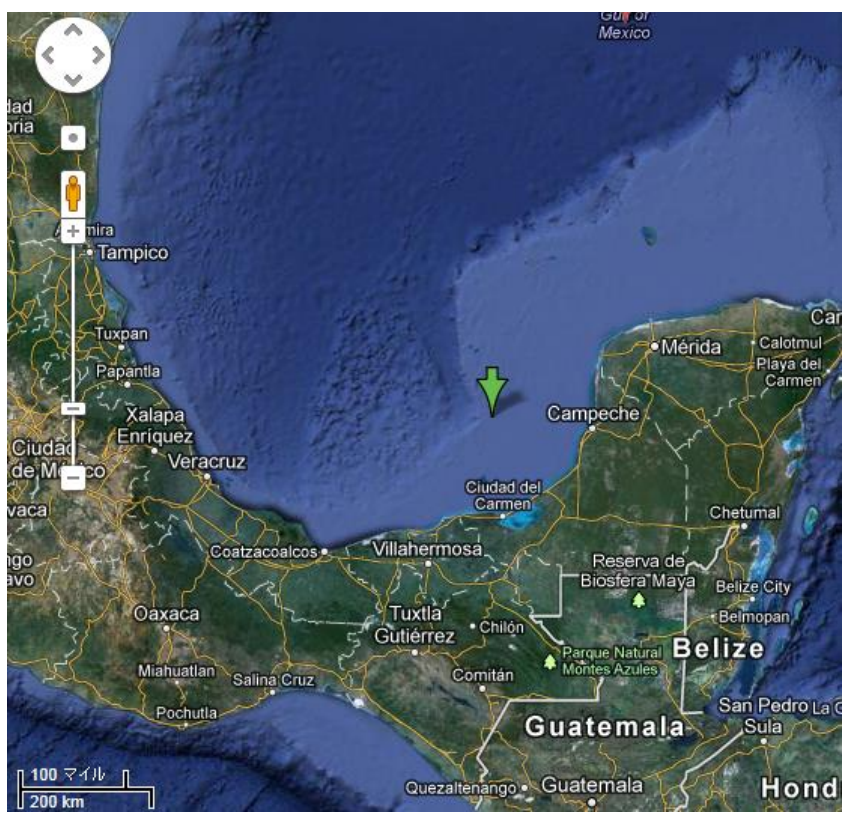

Figure 2. Intensive study areas (Mexican gulf)

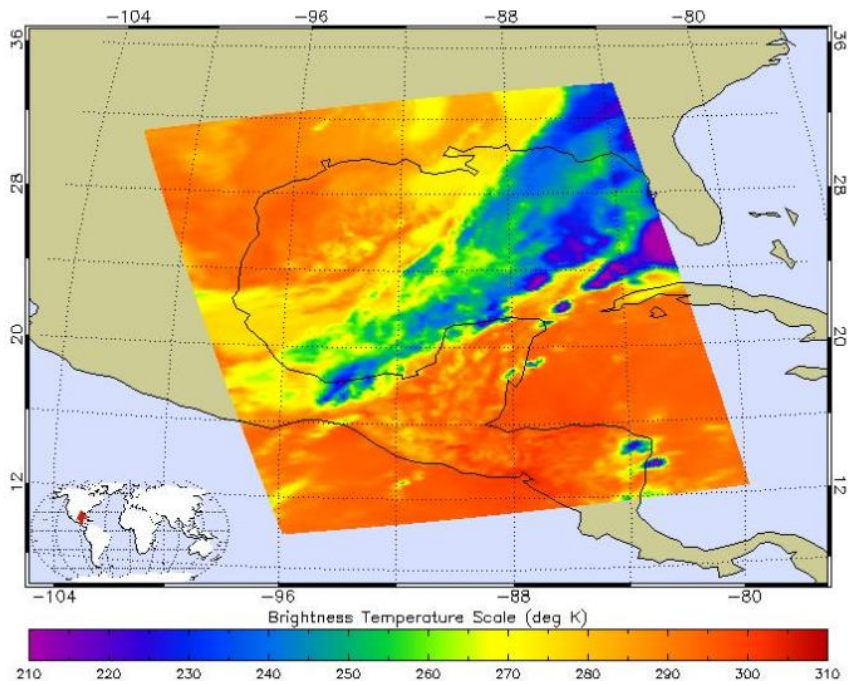

Figure 3. AIRS Level 1B of brightness temperature image

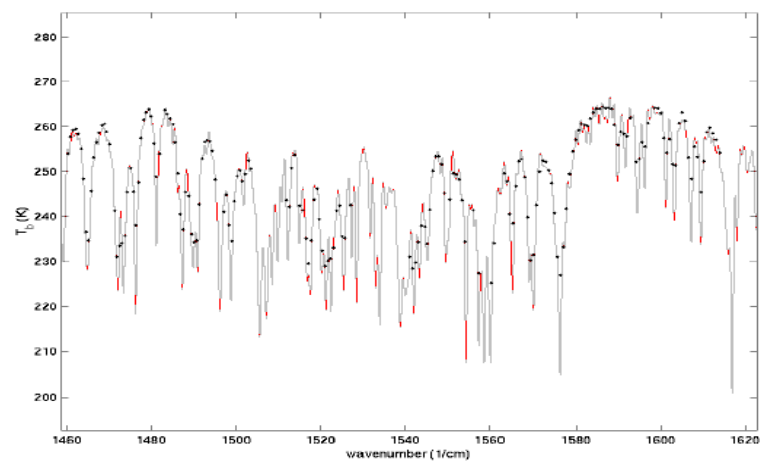

Figure 4. One of the examples of at sensor brightness temperature of AIRS (Water vapor absorption channels of the wave number ranges from 1460 to $1620 \mathrm{~cm}-1)$

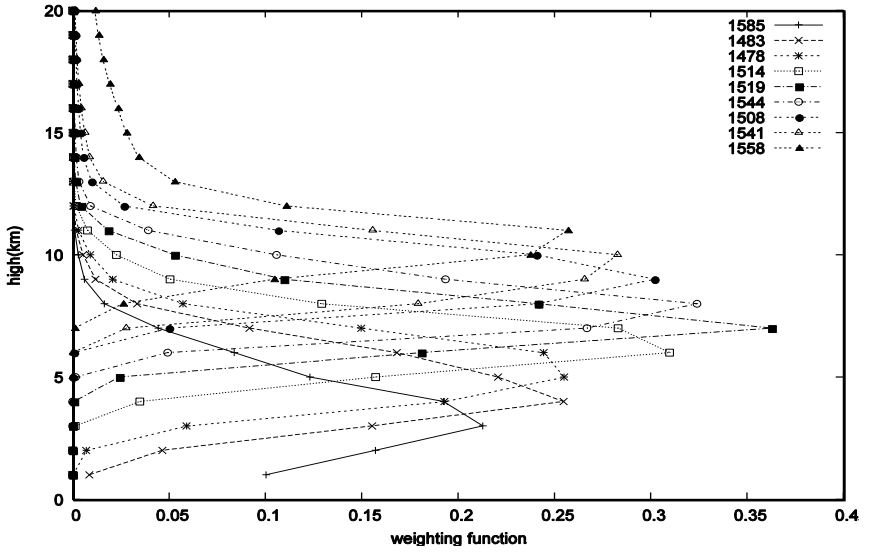

Figure 5. Weighting functions which correspond to the water vapor absorption channels of wave number

\section{Procedure for Water Vapor Profile Retrievals}

Based on the process flow which is shown in Figure 6, water vapor profile is retrieved. First, AIRS channels, wave numbers have to be determined. Then proposed method is applied to the AIRS data as well as MODTRAN derived at sensor brightness temperature.

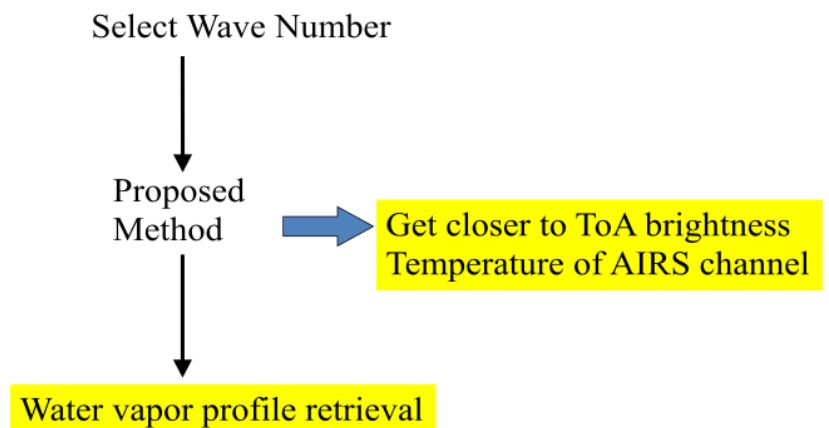

Figure 6. Process flow for water vapor profile retrievals

In this process, initial value of the steepest descent method is given by the brightness temperature derived from linearized inversion. Minimizing the difference between estimated and actual AIRS brightness temperature, water vapor profile is retrieved. Figure 7 shows the difference of brightness temperature between the estimated Top of the Atmosphere: ToA brightness temperature for each AIRS channel of the corresponding wave number for retrieving water vapor profile and actual AIRS data derived brightness temperature at the convergence. The residual error is quite small Convergence condition is set at residual error in below $1 \times 10-8$.

\section{E. Retrieved Water Vapor Profile Based on the Proposed Method}

Retrieved water vapor profile by using the proposed method is shown in Figure 8. AIRS data used for the experiments is acquired on November 16 2002. Also, atmospheric model used for MODTRAN is Mid. Latitude Winter. Therefore, initial value of steepest descent method is close to the water vapor profile of Mid. Latitude Winter of atmospheric model. 
Then the solution is updated to minimize the difference between AIRS derived brightness temperature and estimated ToA brightness temperature derived from MODTRAN.

Initial value is set by the calculated result from the linearized inversion, $\mathrm{K}=\mathrm{B}-1 \mathrm{R}$. The initial value is much closer to the default value of Mid. Latitude Winter model of MODTRAN. Therefore, it seems that the convergence stage of solution seems like a global optimum solution not one of local minima. If the initial value is set with random number, then convergence stage of solution looks like one of local minima, not global optimum solution. It took much longer time in this case while retrieval accuracy is not so good.

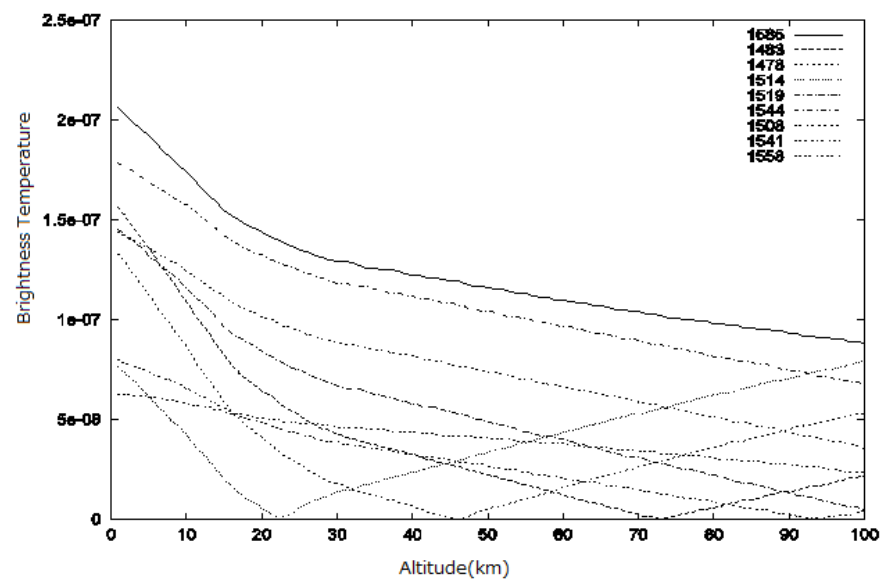

Figure 7. Examples of brightness temperature profile retrievals for the assigned AIRS channels, wave numbers

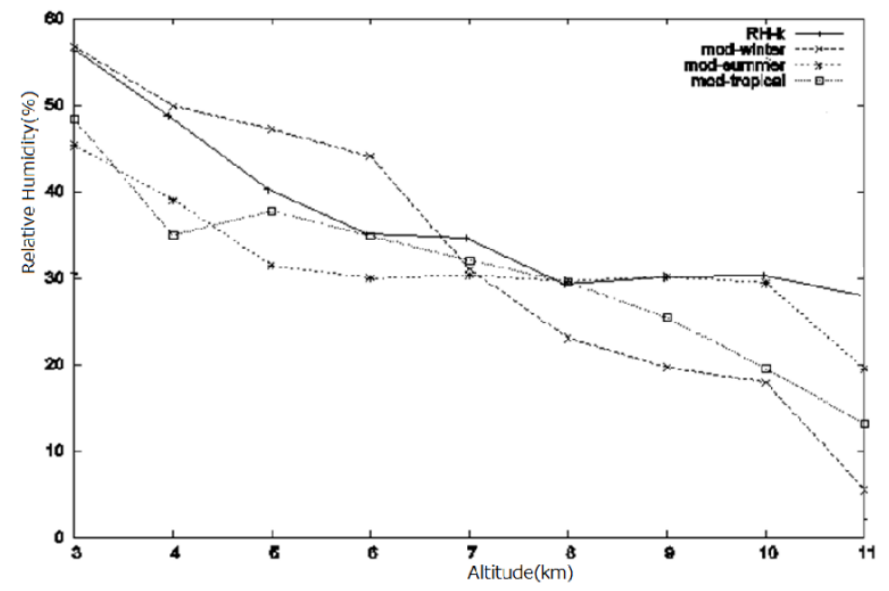

Figure 8. Retrieved relative humidity, water vapor profile and comparison to the default relative humidity profiles for atmospheric model of Mid. Latitude winter, and summer as well as tropic atmosphere

\section{CONCLUSION}

Method for water vapor profile retrievals by means of minimizing difference between estimated and actual brightness temperatures derived from AIRS data and radiative transfer model is proposed. Initial value is determined by linearized radiative transfer equation.
It is found that this initial value determination method makes improvement of estimation accuracy together with reducing convergence time.

Initial value is set by the calculated result from the linearized inversion, $\mathrm{K}=\mathrm{B}-1 \mathrm{R}$. The initial value is much closer to the default value of Mid. Latitude Winter model of MODTRAN. Therefore, it seems that the convergence stage of solution seems like a global optimum solution not one of local minima. If the initial value is set with random number, then convergence stage of solution looks like one of local minima, not global optimum solution. It took much longer time in this case while retrieval accuracy is not so good

\section{ACKNOWLEDGMENT}

The author would like to thank Mr. Noriaki Yamada for his effort to conduct experiments.

\section{REFERENCES}

[1] Kohei Arai, Lecture Note on Remote Sensing, Morikita-Shuppan publishing Co. Ltd, 2004.

[2] NASA/JPL, "AIRS Overview". NASA/JPL. http://airs.jpl.nasa.gov/overview/overview/.

[3] NASA "Aqua and the A-Train". NASA. http://www.nasa.gov/mission_pages/aqua/.

[4] NASA/GSFC "NASA Goddard Earth Sciences Data and Information Services Center". NASA/GSFC. http://disc.gsfc.nasa.gov/AIRS/data_products.shtml.

[5] NASA/JPL "How AIRS Works". NASA/JPL. http://airs.jpl.nasa.gov/technology/how_AIRS_works.

[6] NASA/JPL "NASA/NOAA Announce Major Weather Forecasting Advancement". http://jpl.nasa.gov/news/news.cfm?release=2005-137.

NASA/JPL.

[7] NASA/JPL "New NASA AIRS Data to Aid Weather, Climate Research". http://www.jpl.nasa.gov/news/features.cfm?feature=1424.

[8] Kohei Arai and Naohisa Nakamizo, Water vapor and air-temperature profile estimation with AIRS data based on Levenberg -Marquardt, Abstract of the 50th COSPAR(Committee on Space Research/ICSU) Congress, A 3.1-0086-08,995, Montreal, Canada, July, 2008

[9] Kohei Arai and XingMing Liang, sensitivity analysis for air temperature profile estimation method around the tropopause using simulated AQUA/AIRS data, Advances in Space Research, 43, 3, 845-851, 2009.

\section{AUTHORS PROFILE}

Kohei Arai, He received BS, MS and PhD degrees in 1972, 1974 and 1982, respectively. He was with The Institute for Industrial Science, and Technology of the University of Tokyo from 1974 to 1978 also was with National Space Development Agency of Japan (current JAXA) from 1979 to 1990 . During from 1985 to 1987 , he was with Canada Centre for Remote Sensing as a Post Doctoral Fellow of National Science and Engineering Research Council of Canada. He was appointed professor at Department of Information Science, Saga University in 1990. He was appointed councilor for the Aeronautics and Space related to the Technology Committee of the Ministry of Science and Technology during from 1998 to 2000. He was also appointed councilor of Saga University from 2002 and 2003 followed by an executive councilor of the Remote Sensing Society of Japan for 2003 to 2005 . He is an adjunct professor of University of Arizona, USA since 1998. He also was appointed vice chairman of the Commission "A" of ICSU/COSPAR in 2008. He wrote 30 books and published 332 journal papers. 